\title{
Patterns of oxygen debt repayment in cardiogenic shock patients sustained with extracorporeal life support: a retrospective study
}

Yuri Ganushchak ( $\nabla$ y.ganushchak@mumc.nl )

Maastricht University Medical Centre

Eva Kurniawati

Maastricht University Medical Centre

Iwan van der Horst

Maastricht University Medical Centre

Sander van Kuijk

Maastricht University Medical Centre

\section{Patrick Weerwind}

Maastricht University Medical Centre

Roberto Lorusso

Maastricht University Medical Centre

Jos Maessen

Maastricht University Medical Centre

\section{Research Article}

Keywords: cardiogenic shock, extracorporeal life support, oxygen debt, oxygen debt repayment schedule

Posted Date: January 4th, 2021

DOl: https://doi.org/10.21203/rs.3.rs-130555/v1

License: (9) (i) This work is licensed under a Creative Commons Attribution 4.0 International License.

Read Full License 


\section{Abstract}

Cardiogenic shock is the most frequent shock in cardiac intensive care, and cardiac dysfunction and sever hypoxia are often seen in critically ill patients. Inadequate organ and tissue perfusion and hypoxia result in anaerobic metabolism with hyperlactatemia and oxygen debt accumulation. However, the role of accumulated oxygen debt in course of cardiogenic shock and hypoxia is not clearly described. Here we first described the existence of several patterns of oxygen debt repayment in cardiogenic shock patients maintained by extracorporeal life support system. Oxygen debt was computed from the lactate concentration at five time points, covering the first 26 hours of ECLS. The patterns as the basic pathophysiological processes are independent from the cause of primary insult. The groups of patients classified to the specific patterns differed by survival rate from $51.5 \%$ to only $4.6 \%$. It is very important that initial class does not predetermine the fate and can change in the course of treatment due to 'between clusters migration'. We believe that our finding of patterns of oxygen debt repayment in cardiogenic shock patients may offer a new insights for a more rational, goal-directed treatment of such highly morbid conditions as hypoxia and cardiogenic shock.

\section{Introduction}

Cardiogenic shock is the most frequent type of shock in the cardiac intensive care and cardiac dysfunction is often seen in critically ill patients. ${ }^{1}$ Cardiogenic shock is an emergency involving a complex and highly morbid vicious cycle of cardiac and systemic decompensation. It includes a complex and diverse disorder of end-organ hypoperfusion, hypoxic endothelial damage, acute coagulopathy, and multisystem organ failure. ${ }^{2}$ Inadequate organ and tissue perfusion results in hypoxia and activation of anaerobic metabolism. ${ }^{3-5}$ In addition to the anaerobic glycolysis, adrenergic stimulation due to accompanying severe stress response can increase aerobic lactate production. ${ }^{6,7}$

Elevated arterial lactic acid levels are associated with in-hospital and 30-day mortality rates independently from treatment modus. ${ }^{7-16}$ However, focus on the lactate concentration, and its clearance erodes the understanding of the shock state's major physiological underpinnings. ${ }^{17}$ Lactate concentration is just a metabolic correlate of the pathophysiological process known as oxygen debt. A better understanding of the mechanisms of oxygen debt accumulation and its recovery, also known as repayment, may be a useful guide in treating shock. ${ }^{17,18}$

Originally, Archibald Hill introduced the term 'oxygen debt' in 1928 as the additional oxygen that must be taken into the body after vigorous exercise to restore all systems to their normal states. ${ }^{19}$ In clinical practice, unlike a physiological increase in metabolic requirements during vigorous exercise, the disparity between the metabolic requirements and the level of oxygen delivery is caused by the decrease of oxygen delivery beyond its critical level. ${ }^{17}$ So, oxygen debt is the accumulation of oxygen deficit over time and represents ${ }^{18}$ both the severity of shock and the time spent in the shock state. Animal and clinical studies demonstrated a direct association of total oxygen debt and mortality. ${ }^{18,20,21}$ Furthermore, the severity of 
end-organs and tissue damage increase with prolongation of the period of oxygen debt and in case of failure to repay oxygen debt. ${ }^{18,22}$ So, timely oxidation of unmetabolized substrates (i.e., oxygen debt repayment schedule) is an essential factor determining the severity of ongoing damage and outcome. ${ }^{17}$

Our retrospective study aims to demonstrate the possible differences in the compensation of metabolic disorder patterns caused by cardiogenic shock in terms of oxygen debt in patients supported by extracorporeal life support (ECLS). It also examines the dependence between the treatment outcome of this group of patients and the oxygen debt repayment schedule.

\section{Results}

\subsection{General characteristics}

During the study period, a total of 153 patients were included with an average age of $61.5 \pm 12.3$ years, and a majority of male patients (66\%).

The distribution of the causes of cardiogenic shock in patients sustained by the extracorporeal life support device is presented in Fig. 1a. A total of 106 patients died. The etiology of cardiogenic shock was not associated with six-month mortality in patients sustained by ECLS (Fig. $1 \mathrm{~b}, \mathrm{X}^{2}(8, N=153)=14.53, \mathrm{p}$ $=0.069$ ). Independently from the etiology of cardiogenic shock, $82.4 \%$ of patients received peripheral cannulation, and $14.4 \%$ had central arterial cannulation and peripheral venous. The decision to start ECLS within 8 hours after the event was made in $97.8 \%$ of survivors versus $76.6 \%$ of non-survivors ( $X^{2}(1$, $\mathrm{N}=153)=10.63, \mathrm{p}=0.001$ ). Furthermore, in $41.5 \%$ of cases in the non-survivor group, ECLS was discontinued during the first 24 hour due to prognosis infausta. That is why the duration of ECLS in the group of non-survivors was lower than in group of six-month survivors $(79.0 \pm 93.7$ hours compared to $150.0 \pm 137.9$ hours, respectively, $p<0.0001)$.

The duration of ECLS was associated with six-month survival (150.0 \pm 137.9 hours in survivors group compared to $79.0 \pm 93.7$ hours, respectively, $p<0.0001$. Discontinuation of ECLS during the first 24 hours due to prognosis infausta, was decided in 44 patients (41.5\%) of all patients that died during the sixmonths follow-up period.

\subsection{Cluster analysis}

Two-step cluster analysis based on the oxygen debt amount at the start of ECLS and its value after two hours of support (as a mark of repayment) generated three clusters (Cluster 1, Cluster 2, and Cluster 3, respectively). The $\mathrm{K}$-means cluster procedure repeated at each time interval, as described in the methods section, allowed to trace the changes of oxygen debt and distribution of patients in clusters (Fig. 2). During the first 2 hours of ECLS, the patients in Cluster 1 demonstrated a rise of oxygen debt (1491 \pm $1556 \mathrm{ml}$ pre-ECLS and $2331 \pm 2272 \mathrm{ml}$ after 2 hours of ECLS, $p=0.016$ ) followed by statistically significant decreasing of oxygen debt at each time interval(Fig. 2). We observed a higher prevalence of post-cardiotomy patients in Cluster 1 after initial classification (Fig. 3a, $p=0.002$ ). However, after 2 hours 
of ECLS support, the differences of the distribution by the cause of cardiogenic shock between clusters was not statistically significant (Fig. 3b, $p=0.19$ ). Figure 4 presents the migration of patients between clusters during the first 26 hours of circulatory support. During this time, seven patients from Cluster 1 were successfully weaned and survived for the six-month follow-up period. Migration of patients with more effective oxygen debt repayment to Cluster 1 as well as reverse migration in patients with a delay of repayment increases survival rate in this cluster from $46.1 \%$ observed after two hours of support to $51.5 \%$ after 26 hours of support and is counterbalanced by a decrease in survival rate in Cluster 2 from 26.5$15.8 \%$. Only one patient assigned to Cluster 3 survived for six months. The observed rise of survival rate in Cluster 3 from $4.8 \%$ after two hours of support to $12.5 \%$ was caused by a decrease of the total number of patients in Cluster 3 from 21 to 8 at the end of the 26 hours observation period (Fig. 5a, b).

The frequency of initiation of ECLS during 8 hours after the event in initial clusters did not differ statistically between clusters $(90.6 \%$ in Cluster $1,79.4 \%$ in Cluster 2 , and $86.7 \%$ in Cluster $3, p=0.30$ ).

The MAP in clusters during the observation period is shown in Fig. 6a. The patients assigned to Cluster 3 had lower MAP despite the higher doses of noradrenaline infusion through all study periods (Fig. $6 \mathrm{~b}$ ). The average ECLS flow was $4.2 \pm 0.8 \mathrm{~L} / \mathrm{min}$ in all clusters and did not differ between clusters and time periods.

\section{Discussion}

To our knowledge, this is the first attempt to describe the patterns of tissue perfusion disorder in patients with cardiogenic shock in terms of oxygen debt accumulation and repayment and its impact on shortterm survival.

The initial two-step cluster analysis algorithm based on the total oxygen debt and its dynamic defined three clusters in our study group (Fig. 2). These, with sequential clusterization, exposed three distinguished patterns of initial oxygen debt and its repayment in our patients. The cluster analysis methods used in combination in our study have numerous successful reports that are more and more often appearing in clinical studies. ${ }^{23-26}$ Interested reader can look for principles and algorithms of cluster analysis elsewhere. ${ }^{27}$

It is also well established that accumulated oxygen debt due to a disproportion between the oxidative requirement and the level of oxygen delivery without timely repayment leads to multiple organ failure and mortality. ${ }^{17}$ Maintenance and fast restoration of oxygen delivery sufficient to facilitate adequate cellular metabolism are fundamental in sustaining organ function. The early improvement of tissue perfusion by all means, including mechanical circulatory support ${ }^{7,28-33}$ and rapid decrease or normalization of lactate levels, is critical for survival. ${ }^{7,34-38}$

Our findings support the relevance and positive impact of the early start of ECLS. The ECLS in patients included in our study was initiated during the first 8 hours after insult in $97.8 \%$ of patients who survived a 
six-month observation period compared to $76.4 \%$ of non-survives. However a relatively high number of cases with a start of ECLS withing 8 hours in non-survivors questions the importance of this factor as the primary determinant of the outcome. Furthermore, the distribution of patients with a start of ECLS within 8 hours in clusters was not statistically significant despite a substantial difference in mortality rates. This can be explained by the fact that our study's initial classification was based on the total oxygen debt, which is the accumulation of oxygen deficit over time and embodies both the severity of shock and time spent in the shock state. ${ }^{17,18}$

The total oxygen debt during the first 26 hours of ECLS and its repayment patterns were derived from the serial measurements of lactate concentration at the study time-points. As a quantitative metric, this variable more adequately represents the metabolic state of the patient ${ }^{17}$ than just a lactate concentration in arterial blood. Linear regression equation, used in our study, based on the animal hemorrhagic shock model described by Dieter Rixen et al., 2001. ${ }^{21}$ However, the equivalence of metabolic acidosis and accumulated oxygen debt permits quantification of the severity of the ischemic shock process in both animals and humans. ${ }^{18,39}$ The total oxygen debt was computed as oxygen debt times the bodyweight of a patient. Total oxygen debt states the additional oxygen that must be taken into the body to restore all systems to their normal states. Considering the prognostic importance of lactate clearance and absolute lactate levels ${ }^{40}$, we used oxygen debt at the start and the end of the period as variables for the clustering procedure. So, the clustering procedure was affected by the initial value of debt and its repayment efficiency.

The distribution of patients by the etiology of cardiogenic shock was statistically significant after the initial cluster step. Observed high frequency of post-cardiotomy patients in Cluster 1 with the lowest initial oxygen debt (Fig. 3a) can be explained by continuous observation and treatment of these patients after the end of surgery till the start of ECLS. The concentration of patients with 'Out of Hospital Circulatory Arrest' in Cluster 3 with the highest accumulated oxygen debt (Fig. 3a) could be expected because of the severity of the condition and commonly longer time between insult and starting ECLS. This difference by etiological factor disappears already after 2 hours of support (Fig. 3b). Although we did not find a statically significant relationship between the etiology of cardiogenic shock and six-month survival (Fig. 1b, $p=0.069$ ), the effect of the initial insult and reversibility of myocardial damage is selfexplanatory. However, on the other hand, our data showed that timely and effective oxygen debt repayment could prevent slipping in a vicious cycle of injury, cardiac and systemic decompensation, and further injury and decompensation. The survival rate was significantly higher in patients initially assigned to Cluster 1 (with accumulated oxygen debt around $1500 \mathrm{~mL}$ ), and it even further increased due to the migration of patients in the course of the 26 hours of ECLS observation period.

Patients in Cluster 1 and Cluster 2 had significantly higher mean arterial pressures (Fig. 6a) and required less norepinephrine infusion rates (Fig. 6b) than patients assigned to Cluster 3 . The clusters in our population could be imposed on cardiogenic shock stages defined by the SCAI Consensus Statement Classification. ${ }^{41,42}$ The patients in Cluster 3 most likely had stage E ("Extremis") or refractory cardiogenic 
shock with relatively low mean arterial and not significant in time intervals decreasing (repayment) of oxygen debt despite ECLS and high rate of norepinephrine infusion(Fig. 6a and b). Cluster 2 pulls together patients who most likely had stage D ("Deteriorating") or a mixture of the last three SCAI shock stages. These patients readily migrated to Cluster 1 or Cluster 3 (Fig. 4). Most of our patients were assigned to Cluster 1. Presumably, these patients were in stage $C$ ("Classic") of cardiogenic shock. These patients required a lower infusion rate of norepinephrine for maintaining mean arterial pressure around $70 \mathrm{mmHg}$ (Fig. 6a, b). However, an observed significant elevation of oxygen debt after 2 hours of circulatory support (Fig. 2) suggests further deterioration, but to our belief, rising lactate with increasing total blood flow with the initiation of ECLS could be explained by washout of lactate from previously hypoperfused tissues. ${ }^{43}$ Furthermore, this cluster's patients demonstrated a steady decrease in total oxygen debt during each following time interval until the end of the observation period (Fig. 2). From this point of view, the absence of an oxygen debt rises after 2 hours of support in Cluster 3 is the sign of vasoplegia and washing out of substrates of anaerobic metabolism to the circulation before the start of ECLS. This suggestion is supported by the highest amount of oxygen debt accumulated (Fig. 2) and persisted hypotension despite the highest infusion rate of norepinephrine through the ECLS observation period (Fig. 6a, b).

Yet, the accumulation of total oxygen debt, even more than $15000 \mathrm{ml}$ (Cluster 3), does not mean inevitable death. Timely and effective repayment of oxygen debt can prevent irreversible damage. Four patients migrated from Cluster 3 to Cluster 2 between eight and 14 hours of support (Fig. 4). Two of these patients migrated further to Cluster 1 between 20 and 26 hours of support and survived until the end of the six-month observation period.

We realize that the amount of 'total oxygen debt' computed using linear regression equation based on animal models of hemorrhagic shock can differ from the actual 'debt'. However, the universal character of pathophysiological mechanisms of oxygen debt accumulation and repayment and a robust linear relation of debt with lactate concentration permits us to use this value as an indicator of the severity and duration of the shock. Furthermore, the application of the oxygen debt theory to the cardiogenic shock patients sustained by ECLS combined with cluster analysis allowed us to describe better and understand the patterns of end-organ hypoperfusion recovery and mechanisms of its failure. The failure of massive oxygen debt repayment in the intensive care unit could be related to persisting abnormal microcirculatory perfusion despite normal oxygen delivery values. ${ }^{44}$ However, one of the most challenging problems in the treatment of patients in severe circulatory shock is that the burden of accumulated oxygen debt must be repaid by timely increasing oxygen delivery above baseline levels to restore metabolic function and prevent ongoing organ injury at the cellular level. ${ }^{17,18,20,39}$ Therefore, just returning oxygen delivery to normal basal levels after an accumulation of oxygen debt is insufficient to prevent subsequent organ injury. Mechanical circulatory support devices, especially with peripheral cannulation, cannot cover the required increase of oxygen delivery.

There are several ways to bypass this limitation and should be further investigated., Decreasing a metabolic rate by permissive hypothermia, used with success in trauma patients, ${ }^{45}$ could create a 
necessary excess (relative) oxygen delivery. Another possible way is to limit the duration of exposure to the products of anaerobic metabolism, and concomitant inflammatory respond reaction is high-volume hemofiltration, which is widely used in critically ill patients ${ }^{46,47}$ or zero-balanced ultrafiltration. ${ }^{48}$

The retrospective single-center design has inherent limitations, including missing data and ascertainment bias. The absence of information about the preserved myocardial contractility and cardiac output in patients sustained by ECLS did not allow us to estimate actual oxygen delivery required for the effective repayment of accumulated oxygen debt. Also, the patients with irreversible myocardium damage, patients who died from postoperative complications other than cardiogenic shock, or complications of ECLS were intentionally not excluded from the analysis and might have affected the reported mortality rate.

Concomitant SIRS reactions, as well as resulted in multiple organ failure in patients in cardiogenic shock, are left beyond our investigation.

Application of oxygen debt theory to the cardiogenic shock patients sustained by v-a ECLS combined with cluster analysis uncovers three patterns of oxygen debt repayment. The patterns have an association with six-months mortality. Understanding the pathophysiology of oxygen debt accumulation and its repayment as a basic pathophysiological process independent from the causes of primary insult may offer new insights for a more rational, goal-directed treatment of this highly morbid condition with cardiogenic shock.

\section{Material And Methods}

\subsection{Study population and design}

Between January 2013 and December 2017, all consecutive patients undergoing veno-arterial ECLS (vaECLS) in a single academic center were included retrospectively. No exclusion criteria were applied. Moreover, there was no formal consent applied due to the retrospective nature of the study. The study was approved as a study to which the Medical Research Involving Human Subjects Act (WMO) does not apply and the need for informed consent was waived by the local ethical committee (Medisch-Ethische Toetsingscommissie Academisch Ziekenhuis Maastricht/Universiteit Maastricht, decision METC 2020 1440).

\subsection{Data collection}

The clinical, laboratory, intervention, and outcome data were collected at the treatment time during the support using the Extracorporeal Life Support Organization (ELSO) forms and computer database. Additional information was extracted by reviewing hospital records. The following clinical data were evaluated at the following time points: before ECLS initiation, at 2, 8, 14, 20, and 26 hours of ECLS: ECLS flow, mean arterial pressure (MAP), lactate concentration, and norepinephrine infusion rate.

Oxygen debt was computed from lactate concentration using the linear regression equation. ${ }^{21}$ 
Oxygen debt $(\mathrm{mL} / \mathrm{kg})=-25.26+13.06 \times[$ lactate $](\mathrm{mmol} / \mathrm{L})$ *

*Oxygen debt is only calculated when lactate $\geq 2.0 \mathrm{mmol} / \mathrm{L}$

Total oxygen debt is the result of the multiplication of oxygen debt computed by Eq. 1 at the patient's body weight. The changes of oxygen debt were evaluated in pre-ECLS - 2 hours (T1), 2-8 hours (T2), 814 hours (T3), 14-20 hours (T4), and 20-26 hours (T5) intervals.

\subsection{Study outcomes}

Six-month survival was used as an outcome parameter. The six-month survival was controlled via the Municipal Personal Records Database.

\subsection{Statistical analysis}

The socio-demographic and clinical characteristics were analyzed using descriptive statistics, including percentages for categorical variables and continuous variables, mean \pm standard deviation (SD) in normally distributed parameters.

A two-step cluster analysis algorithm was used for initial stratification. The Z-scores of total oxygen debt before the start of ECLS and after 2 hours of support were selected for detecting primary subgroups of patients with similar patterns of oxygen debt and its repayment dynamics.

Two-steps cluster analysis was performed for the initial separation of patients' sample and generating groups based on similar oxygen debt and its compensation by K-means. The oxygen debt parameters after the $\mathrm{T} 1$ period generated the initial set of centers for the K-means cluster procedure in a sequential period between 2 and 8 hours of support (T2). The algorithm then proceeds by repeating the K-means cluster procedure where the generated cluster centers at the end of each study period were used as initial cluster centers for the next (sequential) period till the end of the 26 hours study period.

The migration of patients between clusters was tracked at each time interval. Analysis of variance (ANOVA) was used to examine the cluster's differences in clinical and laboratory parameters at each time interval. The Pearson chi-square test tested the distribution of patients in clusters by categorical variables. Tests were considered statistically significant at the $95 \%$ confidence interval ( $p$-value $\leq 0.05$ ). Statistical analyses were performed using SPSS version 23 (SPSS Inc., Chicago, IL, USA).

\section{Declarations}

\section{Ethics approval and consent to participate}

The study was approved by medical-ethical approval commission of Maastricht University Medical Centre (METC 2020-1448, 18-06-2020). No written consent was required. 


\section{Consent for publication}

Not applicable.

\section{Availability of data and materials}

Available on request.

\section{Competing interests}

The authors declare that they have no competing interests.

\section{Funding}

this work was not supported by any funding

\section{Authors' contributions}

YG: Conceptualization, Methodology, Writing - original draft;

EK: Data curation, Writing-original draft;

IW: Supervision, Writing - review \& editing;

SK: Methodology, Formal Analysis;

PW: Writing - review \& editing;

RL: Supervision, Writing - review \& editing;

JM: Project administration, Supervision, Writing - review \& editing

All authors read and approved the final manuscript.

\section{References}

1. Berg DD, Bohula EA, van Diepen S, et al. Epidemiology of Shock in Contemporary Cardiac Intensive Care Units. Circ Cardiovasc Qual Outcomes. 2019;12(3):e005618.

2. Bjerkvig CK, Strandenes G, Eliassen HS, et al. "Blood failure" time to view blood as an organ: how oxygen debt contributes to blood failure and its implications for remote damage control resuscitation. Transfusion. 2016;56 Suppl 2:S182-189. 
3. Ronco JJ, Fenwick JC, Tweeddale MG, et al. Identification of the Critical Oxygen Delivery for Anaerobic Metabolism in Critically III Septic and Nonseptic Humans. JAMA. 1993;270(14):17241730.

4. Lazzeri C, Valente S, Chiostri M, Gensini GF. Clinical significance of lactate in acute cardiac patients. World J Cardiol. 2015;7(8):483-489.

5. Bakker J, Postelnicu R, Mukherjee V. Lactate: Where Are We Now? Crit Care Clin. 2020;36(1):115-124.

6. Garcia-Alvarez M, Marik P, Bellomo R. Stress hyperlactataemia: present understanding and controversy. Lancet Diabetes Endocrinol. 2014;2(4):339-347.

7. van Diepen S, Katz JN, Albert NM, et al. Contemporary Management of Cardiogenic Shock: A Scientific Statement From the American Heart Association. Circulation. 2017;136(16):e232-e268.

8. Rixen D, Siegel JH. Metabolic correlates of oxygen debt predict posttrauma early acute respiratory distress syndrome and the related cytokine response. J Trauma. 2000;49(3):392-403.

9. Lindholm MG, Hongisto M, Lassus J, et al. Serum Lactate and A Relative Change in Lactate as Predictors of Mortality in Patients with Cardiogenic Shock - Results from The Cardshock Study. Shock. 2019.

10. Hayiroglu MI, Keskin M, Uzun AO, et al. Predictors of In-Hospital Mortality in Patients With STSegment Elevation Myocardial Infarction Complicated With Cardiogenic Shock. Heart Lung Circ. 2019;28(2):237-244.

11. Gjesdal G, Braun OO, Smith JG, Schersten F, Tyden P. Blood lactate is a predictor of short-term mortality in patients with myocardial infarction complicated by heart failure but without cardiogenic shock. BMC Cardiovasc Disord. 2018;18(1):8.

12. Pabst D, Foy AJ, Peterson B, Soleimani B, Brehm CE. Predicting Survival in Patients Treated With Extracorporeal Membrane Oxygenation After Myocardial Infarction. Crit Care Med. 2018;46(5):e359e363.

13. Ro SK, Kim WK, Lim JY, Yoo JS, Hong SB, Kim JB. Extracorporeal life support for adults with refractory septic shock. J Thorac Cardiovasc Surg. 2018.

14. Davierwala PM, Leontyev S, Verevkin A, et al. Temporal Trends in Predictors of Early and Late Mortality After Emergency Coronary Artery Bypass Grafting for Cardiogenic Shock Complicating Acute Myocardial Infarction. Circulation. 2016;134(17):1224-1237.

15. Valente S, Lazzeri C, Vecchio S, et al. Predictors of in-hospital mortality after percutaneous coronary intervention for cardiogenic shock. Int J Cardiol. 2007;114(2):176-182.

16. Hernandez G, Bellomo R, Bakker J. The ten pitfalls of lactate clearance in sepsis. Intensive Care Med. 2019;45(1):82-85.

17. Barbee RW, Reynolds PS, Ward KR. Assessing shock resuscitation strategies by oxygen debt repayment. Shock. 2010;33(2):113-122.

18. Rixen D, Siegel JH. Bench-to-bedside review: oxygen debt and its metabolic correlates as quantifiers of the severity of hemorrhagic and post-traumatic shock. Crit Care. 2005;9(5):441-453. 
19. Hill AV. The diffusion of oxygen and lactic acid through tissues. Proceedings of the Royal Society of London Series B, Containing Papers of a Biological Character. 1928;104(728):39.

20. Dunham CM, Siegel JH, Weireter $L$, et al. Oxygen debt and metabolic acidemia as quantitative predictors of mortality and the severity of the ischemic insult in hemorrhagic shock. Crit Care Med. 1991;19(2):231-243.

21. Rixen $D$, Raum M, Holzgraefe B, et al. A pig hemorrhagic shock model: oxygen debt and metabolic acidemia as indicators of severity. Shock. 2001;16(3):239-244.

22. Shoemaker WC, Appel PL, Kram HB. Tissue oxygen debt as a determinant of lethal and nonlethal postoperative organ failure. Crit Care Med. 1988;16(11):1117-1120.

23. Ganushchak YM, Fransen EJ, Visser C, De Jong DS, Maessen JG. Neurological complications after coronary artery bypass grafting related to the performance of cardiopulmonary bypass. Chest. 2004;125(6):2196-2205.

24. Jain AK. Data clustering: 50 years beyond K-means. Pattern Recognition Letters. 2010;31(8):651-666.

25. van Giessen A, de Wit GA, Smit HA, et al. Patient selection for cardiac surgery: Time to consider subgroups within risk categories? International Journal of Cardiology. 2016;203:1103-1108.

26. Castela Forte J, Perner A, van der Horst ICC. The use of clustering algorithms in critical care research to unravel patient heterogeneity. Intensive Care Medicine. 2019;45(7):1025-1028.

27. Han J, Kamber M, Pei J. Data Mining: Concepts and Techniques. 3d ed. San Francisco, CA, USA: Morgan Kaufmann Publishers Inc. ; 2011.

28. den Uil CA, Maat AP, Lagrand WK, et al. Mechanical circulatory support devices improve tissue perfusion in patients with end-stage heart failure or cardiogenic shock. $J$ Heart Lung Transplant. 2009;28(9):906-911.

29. Werdan K, Gielen S, Ebelt H, Hochman JS. Mechanical circulatory support in cardiogenic shock. Eur Heart J. 2014;35(3):156-167.

30. Mungan I, Kazancı D, Bektaş Ş, Ademoglu D, Turan S. Does lactate clearance prognosticates outcomes in ECMO therapy: a retrospective observational study. BMC anesthesiology. 2018;18(1):152-152.

31. Jones TL, Nakamura K, McCabe JM. Cardiogenic shock: evolving definitions and future directions in management. Open Heart. 2019;6(1):e000960.

32. Marin F, Pighi M, Pesarini G, Piccoli A, Ribichini F. Devices for mechanical circulatory support and strategies for their management in cardiogenic shock. Kardiol Pol. 2019;77(6):589-595.

33. Berg DD, Barnett CF, Kenigsberg BB, et al. Clinical Practice Patterns in Temporary Mechanical Circulatory Support for Shock in the Critical Care Cardiology Trials Network (CCCTN) Registry. Circ Heart Fail. 2019;12(11):e006635.

34. den Uil CA, Lagrand WK, Valk SD, Spronk PE, Simoons ML. Management of cardiogenic shock: focus on tissue perfusion. Curr Prob/ Cardiol. 2009;34(8):330-349. 
35. Donnino MW, Miller J, Goyal N, et al. Effective lactate clearance is associated with improved outcome in post-cardiac arrest patients. Resuscitation. 2007;75(2):229-234.

36. Jansen TC, van Bommel J, Schoonderbeek FJ, et al. Early lactate-guided therapy in intensive care unit patients: a multicenter, open-label, randomized controlled trial. Am J Respir Crit Care Med. 2010;182(6):752-761.

37. Vincent J-L, Quintairos e Silva A, Couto L, Taccone FS. The value of blood lactate kinetics in critically ill patients: a systematic review. Critical Care. 2016;20(1):257.

38. Hernandez G, Ospina-Tascon GA, Damiani LP, et al. Effect of a Resuscitation Strategy Targeting Peripheral Perfusion Status vs Serum Lactate Levels on 28-Day Mortality Among Patients With Septic Shock: The ANDROMEDA-SHOCK Randomized Clinical Trial. JAMA. 2019;321(7):654-664.

39. Siegel JH, Fabian M, Smith JA, et al. Oxygen debt criteria quantify the effectiveness of early partial resuscitation after hypovolemic hemorrhagic shock. J Trauma. 2003;54(5):862-880; discussion 880.

40. Staudacher D, Supady A, Biever P, et al. Comprehensive Analysis Of Absolute Lactate Levels and Lactate Clearance in VA-ECMO Patients \&\#x2013; Impact on Survival. Resuscitation. 2019;142:e12.

41. Baran DA, Grines CL, Bailey S, et al. SCAI clinical expert consensus statement on the classification of cardiogenic shock: This document was endorsed by the American College of Cardiology (ACC), the American Heart Association (AHA), the Society of Critical Care Medicine (SCCM), and the Society of Thoracic Surgeons (STS) in April 2019. Catheter Cardiovasc Interv. 2019;94(1):29-37.

42. Jentzer JC, van Diepen S, Barsness GW, et al. Cardiogenic Shock Classification to Predict Mortality in the Cardiac Intensive Care Unit. Journal of the American College of Cardiology. 2019;74(17):21172128.

43. Ganushchak YM, Maessen JG, de Jong DS. The oxygen debt during routine cardiac surgery: illusion or reality? Perfusion. 2002;17(3):167-173.

44. Ince $\mathrm{C}$. Hemodynamic coherence and the rationale for monitoring the microcirculation. Crit Care. 2015;19 Suppl 3:S8.

45. Aggen D, Azeem M, Labana S, et al. How Cold Is Cold Enough? Retrospective Review of Therapeutic Hypothermia Outcomes and Protocol Revision in a Community-Based Hospital. CHEST. 2015;148(4):241A.

46. Junhai Z, Beibei C, Jing Y, Li L. Effect of High-Volume Hemofiltration in Critically III Patients: A Systematic Review and Meta-Analysis. Medical science monitor : international medical journal of experimental and clinical research. 2019;25:3964-3975.

47. Luo Y, Sun $G$, Zheng $C$, et al. Effect of high-volume hemofiltration on mortality in critically ill patients: A PRISMA-compliant systematic review and meta-analysis. Medicine. 2018;97(38):e12406.

48. Mick S, Hilberath JN, Davidson MJ, Fitzgerald D. Zero balance ultrafiltration for the correction of acute acidosis after a period of prolonged deep hypothermic circulatory arrest. Perfusion. 2012;27(1):9-11.

\section{Figures}




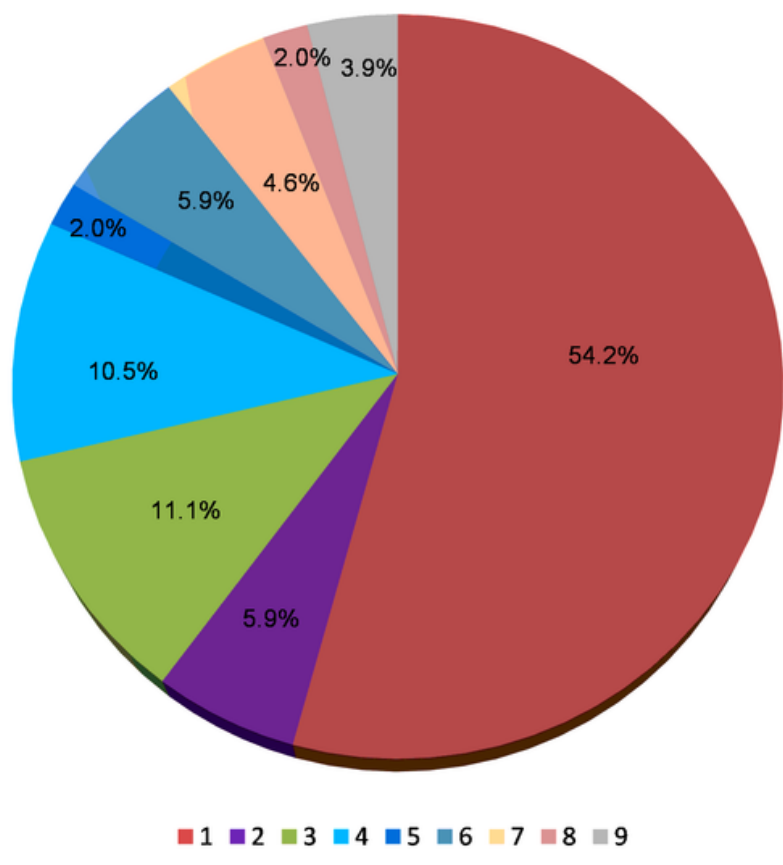

a)

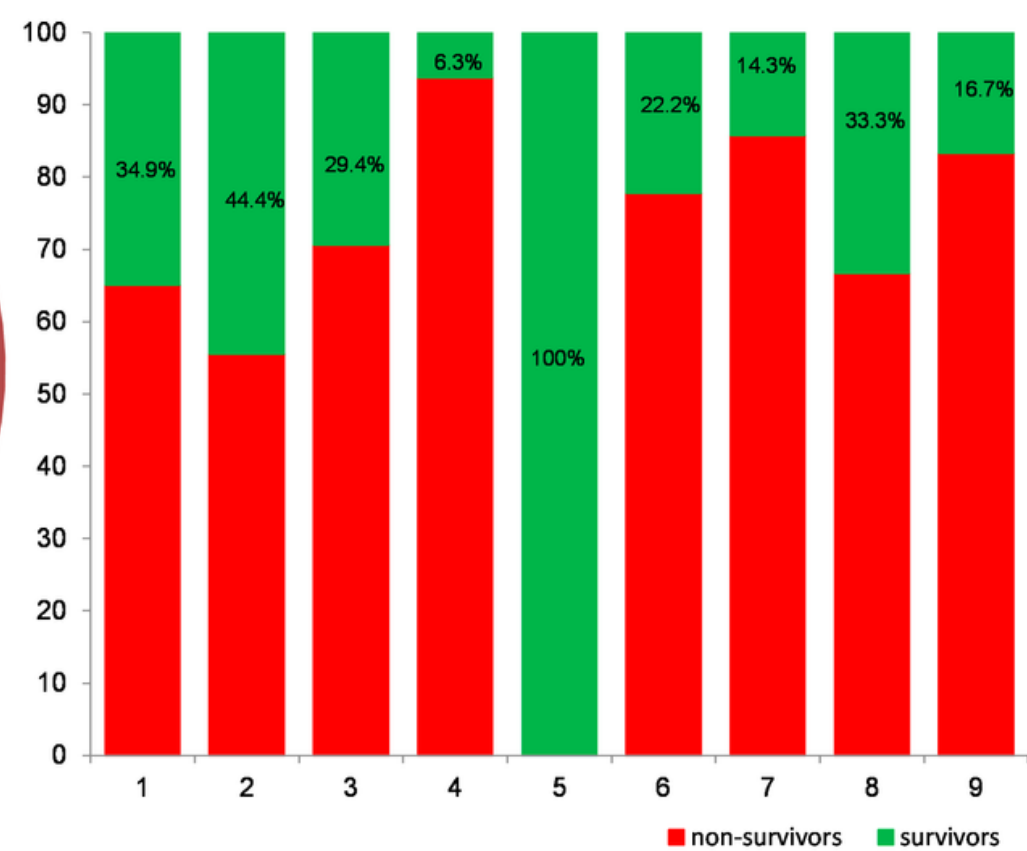

b)

1 - postcardiotomy; 2 - myocarditis; 3 - myocardial infarction; 4 - OHCA; 5 - arrhythmia; 6 - pulmonary embolism; 7 - postPTCA; 8 - cardiomyopathy; 9 - other.

\section{Figure 1}

a) Causes of cardiogenic shock in the patient cohort; (b) survival rate by etiology of cardiogenic shock ( $\chi 2$ $(8, N=153)=14.532, p>0.05)$. 


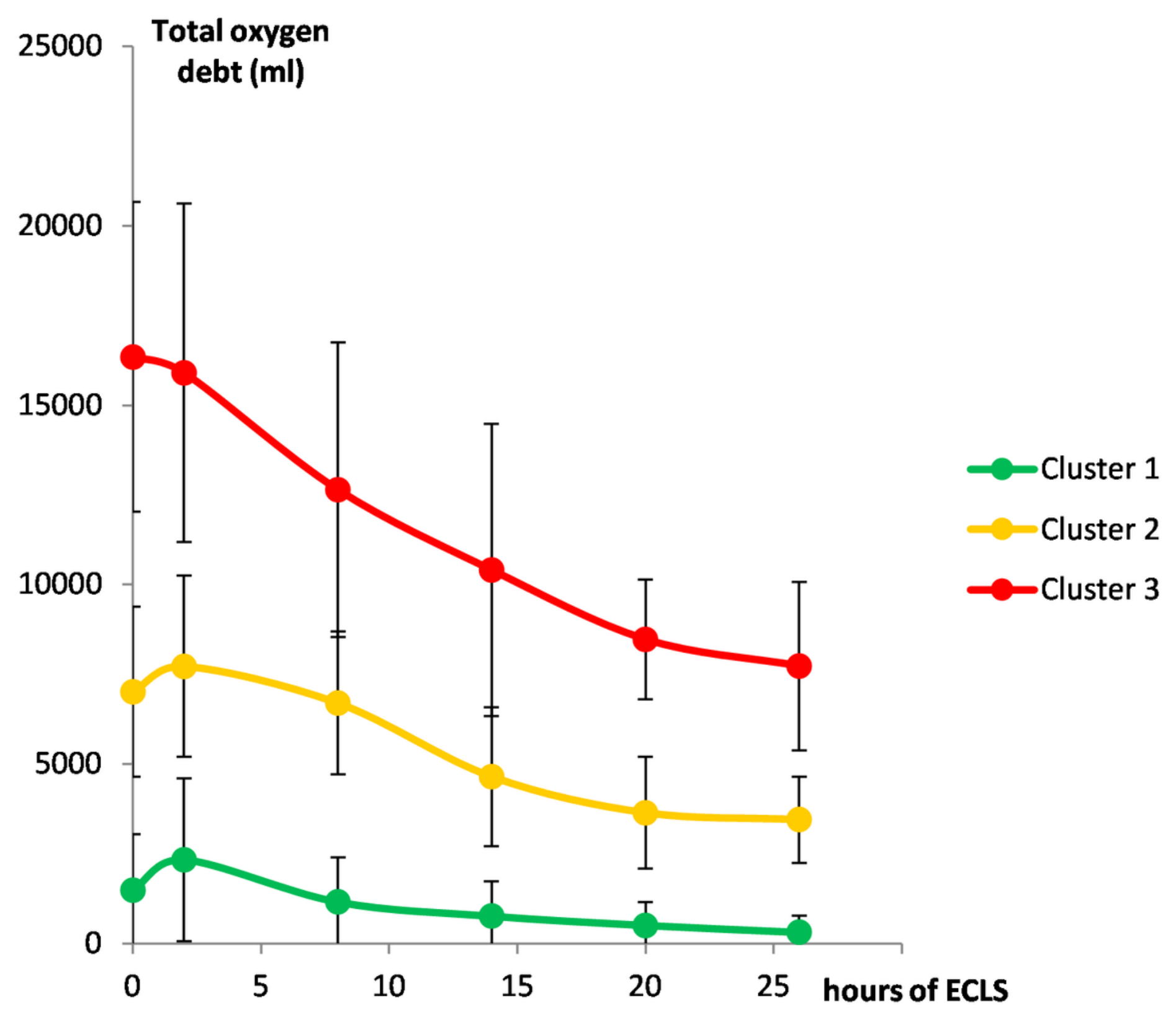

Figure 2

Clusters centers shift in five time periods. Statistically significant shift of clusters centers at T1 $0-2$ hours: cluster $1-F(1,126)=5.95, p=0.016$; at T2 $2-8$ hours: cluster $1-F(1,136)=14.64, p<0.0001$, and cluster $3-F(1,36)=5.04, p=0.031 ; T 38-14$ hours: cluster $1-F(1,145)=4.59, p=0.034$, and cluster $2-F(1,61)=17.09, p<0.0001 ; T 414-20$ hours: cluster $2-F(1,55)=4.63, p=0.036 ;$ T5 $20-26$ no significant changes in clusters. 

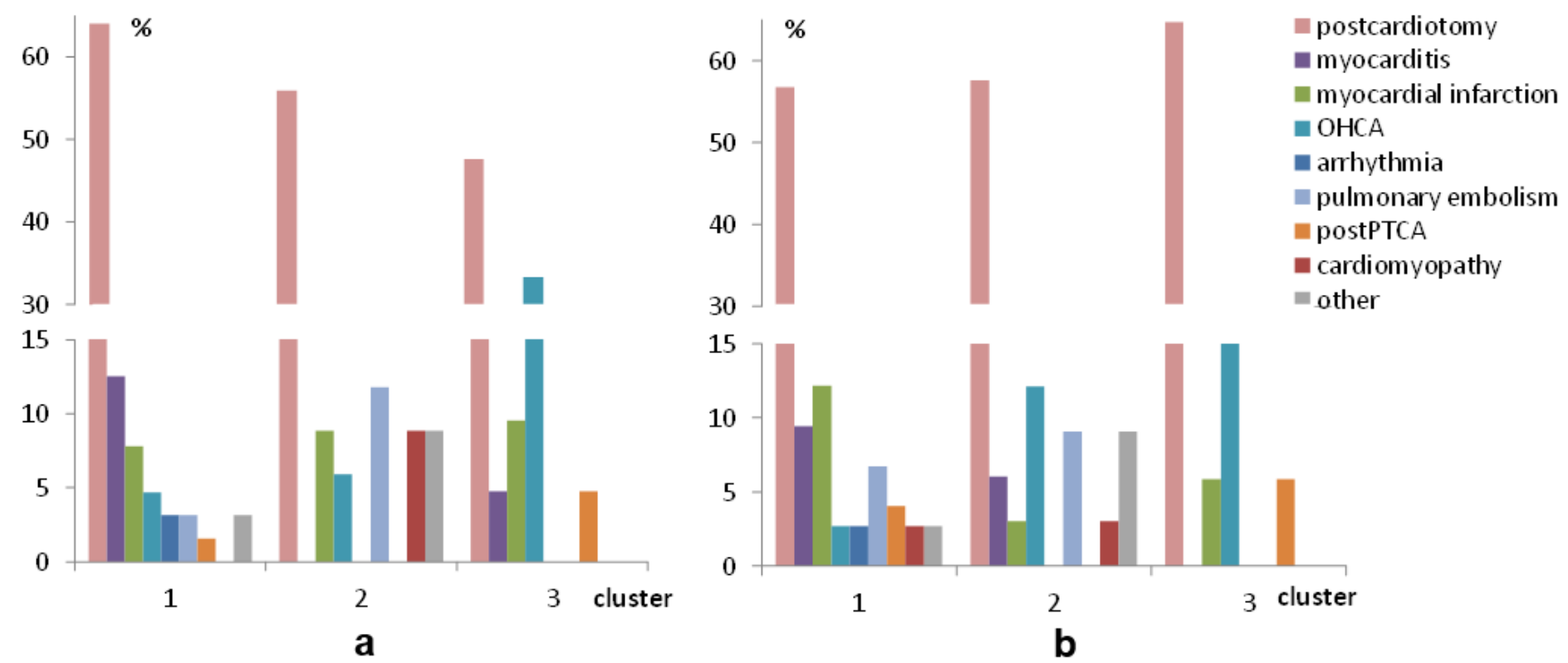

\section{Figure 3}

Distribution of patients by etiology of cardiogenic shock in clusters. (a) Initial classification. There is a significant differences in distribution patients by etiology of CS in clusters $(X 2(16, N=119)=37.756, p=$ $0.002)$ which disappears already after 2 hours $(b)$ of support $(\chi 2(16, N=124)=20.642, p>0.05)$. OHCA: out of hospital cardiac arrest; PTCA: percutaneous coronary angioplasty.

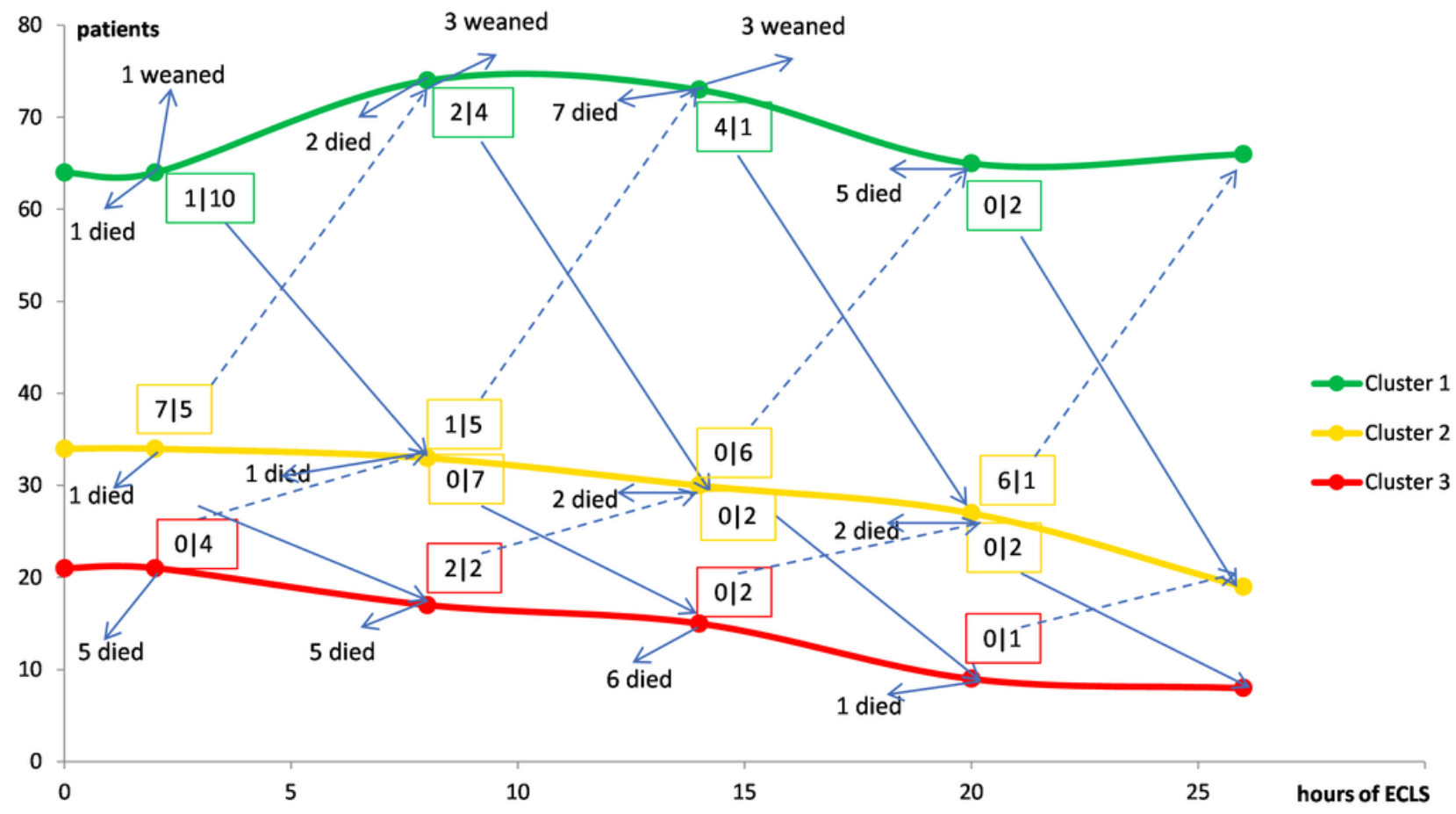

Figure 4 
Patients' migration in the course of extracorporeal life support. In colored text boxes is presented the number of migrated patients' survivors | non-survivors. The migration of patients took place through all 26 hours of observation period. Only patients from the first cluster were successfully weaned during first 26 hours of support and survived for the 6 month.

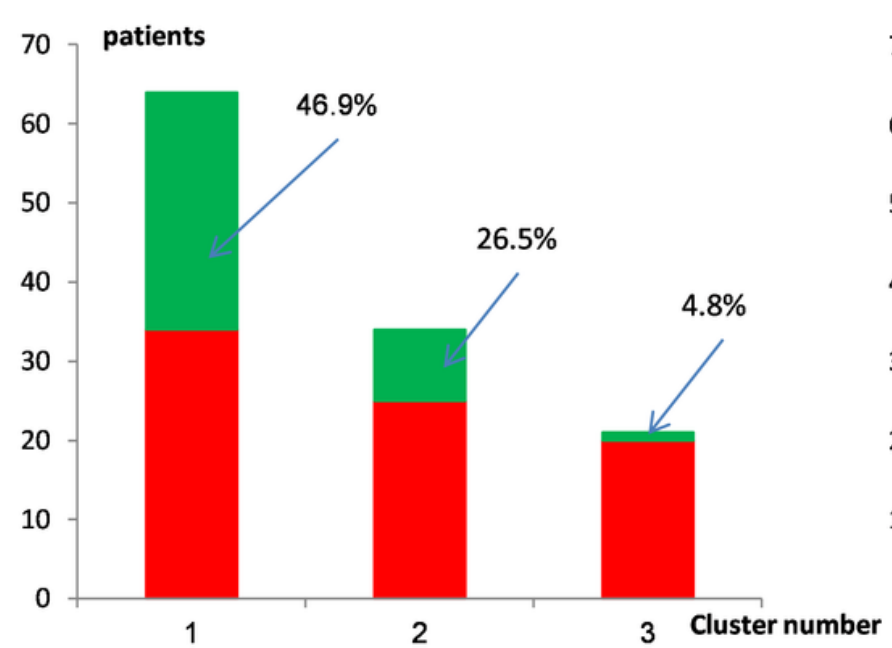

a)

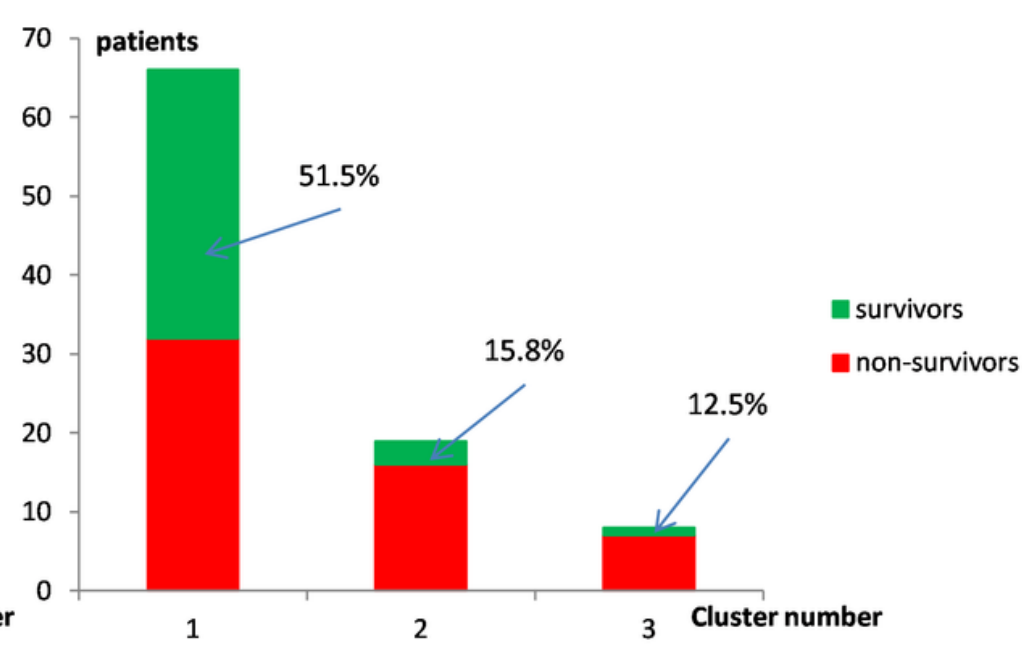

b)

\section{Figure 5}

Six month survival in clusters. Bars represent total number of patients in cluster. Labels are the 6 month survival rate in patients assigned to clusters initially (a) and at the end of the observation period (b). The distribution survivors | non-survivors in clusters was statistically significant through all ECLS observation periods. At the shown examples $\chi 2(2, N=119)=13.66, p<0.0001$ after initial classification (a) and $\chi 2$ $(2, \mathrm{~N}=93)=10.71, \mathrm{p}=0.005$ after 26 hours of support $(\mathrm{b})$. 

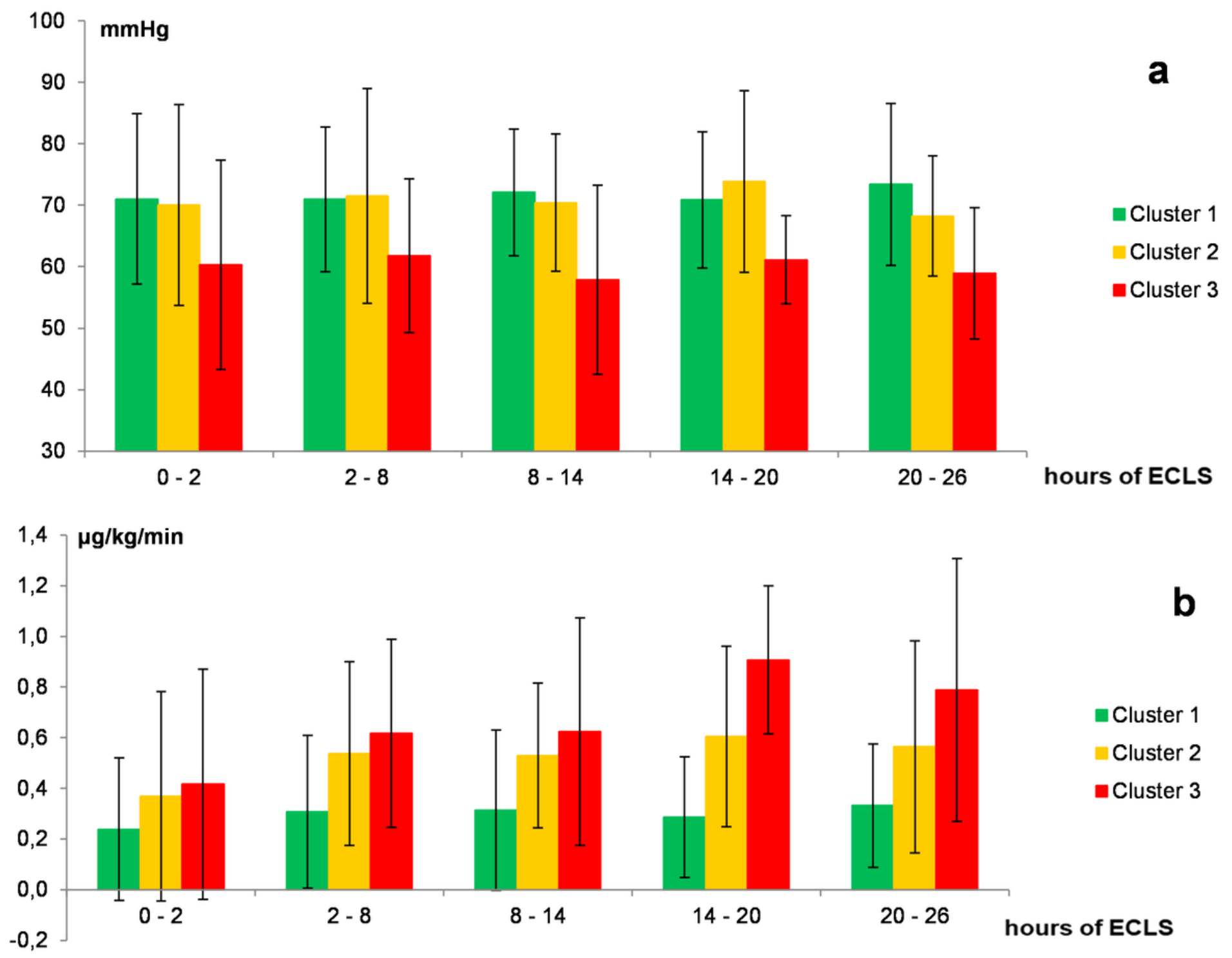

Figure 6

a) mean arterial pressure in clusters in time periods. The mean arterial pressure significantly differed in clusters in timepoints: T2 (2 - 8 hours) $-F(2,118)=3.31, p=0.04 ;$ T3 $(8-14$ hours $)-F(2,112)=8,89, p$ $<0.0001$; T4 (14 - 20 hours) $-F(2,97)=3.85, p=0.025$; period of ECLS; b) norepinephrine infusion rate in clusters in time periods. The infusion rate significantly differed in clusters in timepoints: T2 (2 - 8 hours) $-F(2,109)=7.68, p=0.001 ; T 4(14-20$ hours $)-F(2,93)=10.29, p<0.0001 ; T 5(20-26$ hours $)-F(1,87)$ $=13.05, \mathrm{p}<0.0001$. 